\title{
Advances in Automatic Software Verification: SV-COMP 2020
}

\author{
Dirk Beyer (D) \\ LMU Munich, Germany
}

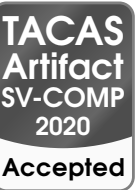

\begin{abstract}
This report describes the 2020 Competition on Software Verification (SV-COMP), the $9^{\text {th }}$ edition of a series of comparative evaluations of fully automatic software verifiers for $\mathrm{C}$ and Java programs. The competition provides a snapshot of the current state of the art in the area, and has a strong focus on replicability of its results. The competition was based on 11052 verification tasks for C programs and 416 verification tasks for Java programs. Each verification task consisted of a program and a property (reachability, memory safety, overflows, termination). SV-COMP 2020 had 28 participating verification systems from 11 countries.
\end{abstract}

Keywords: Formal Verification · Program Analysis · Competition

\section{Introduction}

The Competition on Software Verification (SV-COMP) serves as the showcase of the state of the art in the area of automatic software verification. SV-COMP 2020 is the $9^{\text {th }}$ edition of the competition and presents an overview of the currently achieved results by tool implementations that are based on the most recent ideas, concepts, and algorithms for fully automatic verification. This competition report describes the (updated) rules and definitions, presents the competition results, and discusses some interesting facts about the execution of the competition experiments. The competition measures its own success by evaluating whether the objectives of the competition were achieved. To the objectives discussed earlier (1-4 [14]) we add two further objectives that deserve mentioning (5-6):

1. provide an overview of the state of the art in software-verification technology and increase visibility of the most recent software verifiers,

2. establish a repository of software-verification tasks that is publicly available for free use as standard benchmark suite for evaluating verification software,

3. establish standards that make it possible to compare different verification tools, including a property language and formats for the results,

4. accelerate the transfer of new verification technology to industrial practice by identifying the strengths of the various verifiers on a diverse set of tasks,

5. educate $\mathrm{PhD}$ students and others on performing replicable benchmarking, packaging tools, and running robust and accurate research experiments, and

6. provide research teams that do not have sufficient computing resources with the opportunity to obtain experimental results on large benchmark sets.

(C) The Author(s) 2020

A. Biere and D. Parker (Eds.): TACAS 2020, LNCS 12079, pp. 347-367, 2020.

https://doi.org/10.1007/978-3-030-45237-7_21 
We now discuss the outcome of SV-COMP 2020 with respect to these objectives: (1) There were 28 participating software systems from 11 countries, using many different technologies (cf. Table 6). SV-COMP is considered an important event in the verification community. (2) The sv-benchmarks repository is considered one of the largest and most diverse collections of verification tasks in $\mathrm{C}$ and Java. The community dedicates a lot of maintenance effort, as the issue tracker ${ }^{1}$ and the pull requests ${ }^{2}$ on GitHub show. (3) SV-COMP has established a format for defining verification tasks, a standard specification language, and a set of functions to express non-deterministic values. Verification results are validated using verification witnesses and six different validators. (4) We received positive feedback from industry, reporting that it is helpful to look up the newest and best available verification tools, regarding the categories of interest. There are several participating systems from industry since 2017. (5) Participating in SV-COMP is also a challenge because the entry requirements are strict: the tools have to be packaged such that all necessary non-standard components are contained, the tools need to provide meaningful log output, the tool parameters have to be specified in the BENCHEXEC benchmark-definition format, and a tool-info module needs to be implemented. All experiments are required to be fully replicable. It is a motivating experience to observe the learning of first-time participants. (6) Running large-scale performance experiments requires an infrastructure with considerable computing resources — which are not necessarily available to all tool developers. Through this competition and the preruns, the participants get the opportunity to repeatedly run experiments on the full benchmark set of verification tasks of the competition. The preruns and final run sum up to over one million verification runs and ten million witness-validation runs.

Related Competitions. It is well-understood that competitions are an important evaluation method, and there are many other competitions in the field of formal methods. The TOOLympics ${ }^{3}$ [7] event in 2019 (part of the 25-years-ofTACAS celebration) presented 16 competitions in the area. Most closely related are the competitions RERS ${ }^{4}$ [45] and VerifyThis ${ }^{5}$ [46]. While SV-COMP ${ }^{6}$ performs replicable experiments in a controlled environment (dedicated resources, resource limits), the RERS Challenges give more room for exploring combinations of interactive with automatic approaches without limits on the resources, and the VerifyThis Competition focuses on evaluating approaches and ideas rather than on fully automatic verification.

Large benchmark collections are extremely important to make approaches comparable and to agree on what constitutes interesting problems to solve. There are other large benchmark collections as well (e.g., by SPEC ${ }^{7}$ ), but the

\footnotetext{
1 https://github.com/sosy-lab/sv-benchmarks/issues

2 https://github.com/sosy-lab/sv-benchmarks/pulls

3 https://tacas.info/toolympics.php

4 http://rers-challenge.org

5 http://etaps2016.verifythis.org

6 https://sv-comp.sosy-lab.org

7 https://www.spec.org
} 
sv-benchmarks suite ${ }^{8}$ is (a) free of charge, and (b) tailored to the state of the art in software verification. Benchmark repositories of various competitions and challenges also contribute to each other. For example, the sv-benchmarks suite contains programs that were originally used in $\mathrm{RERS}^{9}$, in termCOMP ${ }^{10}$, and in VerifyThis ${ }^{11}$. There is a flow of benchmarks in the other direction as well: The competition SMT-COMP [32] uses SMT formulas that were generated from programs of the sv-benchmarks collection. For example, the $k$-induction engine of CPACHECKER was used to generate more than 1000 SMT formulas for the quantifier-free theory of arrays and bit-vectors $\left(\mathrm{QF} \_\mathrm{ABV}\right)^{12}$.

\section{Organization, Definitions, Formats, and Rules}

Procedure. SV-COMP 2020's overall organization did not change in comparison to the earlier editions $[8,9,10,11,12,13,14]$. SV-COMP is an open competition, where all verification tasks are known before the submission of the participating verifiers, which is necessary due to the complexity of the $\mathrm{C}$ language. During the benchmark submission phase, new verification tasks were collected, classified, and added to the existing benchmark suite (i.e., SV-COMP uses an accumulating benchmark suite), during the training phase, the teams inspected the verification tasks and trained their verifiers (also, the verification tasks received fixes and quality improvement), and during the evaluation phase, verification runs were preformed with all competition candidates, and the system descriptions and archives were reviewed by the competition jury. The participants received the results of their verifier directly via e-mail, and after a few days of inspection, the results were publicly announced on the competition web site. The Competition Jury consisted again of the chair and one member of each participating team. Team representatives of the jury are listed in Table 5 .

Qualification and License Requirements. As a new feature in SV-COMP 2020, a rule was introduced that allows the organizer to reuse systems that participated in previous years, and to enter new systems, provided that the developers were given the chance to contribute a submission themselves (both options were not used this time). Starting 2018, SV-COMP required that the verifier must be publicly available for download and has a license that

(i) allows replication and evaluation by anybody (including results publication),

(ii) does not restrict the usage of the verifier output (log files, witnesses), and

(iii) allows any kind of (re-)distribution of the unmodified verifier archive.

\footnotetext{
8 https://github.com/sosy-lab/sv-benchmarks

9 https://github.com/sosy-lab/sv-benchmarks/blob/svcomp20/c/eca-rers2012/README.txt

10 https://github.com/sosy-lab/sv-benchmarks/blob/svcomp20/c/termination-restricted-15/ README.txt

11 https://github.com/sosy-lab/sv-benchmarks/blob/svcomp20/c/verifythis/README.txt

12 https://clc-gitlab.cs.uiowa.edu:2443/SMT-LIB-benchmarks-inc/QF_ABV/tree/master/ 20190307-CPAchecker_kInduction-SoSy_Lab
} 


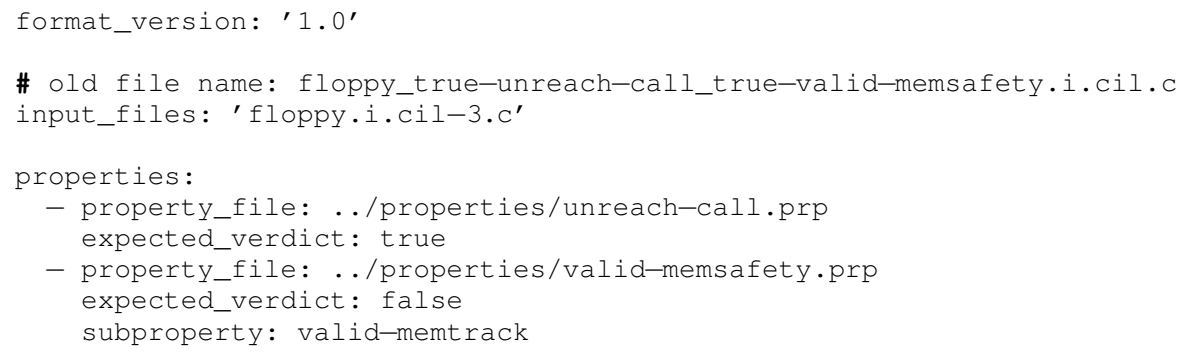

Fig. 1: Example task definition for program floppy.i.cil-3.c

Validation of Results. The validation of the results based on verification witnesses $[19,20]$ was done as in previous years (2017-2019), mandatory for both answers TRUE or FALSE. A few categories were excluded from validation if the validators did not sufficiently support a certain kind of program or property. Two new validators participated in SV-COMP 2020: Nitwit [66] and Meta Val [25].

Verification Tasks - Explicit Task-Definition Files. The notion of verification tasks did not change and we refer to previous reports for more details $[10,13]$. We developed a new format for task definitions that was used for the Java category already in SV-COMP 2019. Technically, we need a verification task (a pair of a program and a specification to verify) to feed as input to the verifier, and an expected result against which we check the answer that the verifier returns. Previously, the above-mentioned three components were specified in the file name of the program; now all the information is stored in an extra file that contains a structured definition of the verification tasks for a program. For each program, the repository contains the program file and a task-definition file. Consider an example program that is available under the name floppy.i.cil-3.c: This program comes now with its task-definition file floppy.i.cil-3.yml. Figure 1 shows this task definition. The new format was used in SV-COMP 2019 for the Java category [14] and in the competition on software testing, Test-Comp 2019 [15].

The task definition uses the YAML format as underlying structured data format. It contains a version id of the format (line 1) and can contain comments (line 3). The field input_files specifies the input program (example: 'floppy.i.cil-3.c'), which is either one file or a list of files. The field properties lists all properties of the specification for this program. Each property has a field property_file that specifies the property file (example: ../properties/unreach-call.prp) and a field expected_verdict that specifies the expected result (example: true).

Categories, Properties, Scoring Schema, and Ranking. The categories are listed in Tables 7 and 8 and described in detail on the competition web site. ${ }^{13}$ Figure 2 shows the category composition. For the definition of the properties and the property format, we refer to the 2015 competition report [11]. All specifications are available in the directory c/properties/ of the benchmark

13 https://sv-comp.sosy-lab.org/2020/benchmarks.php 


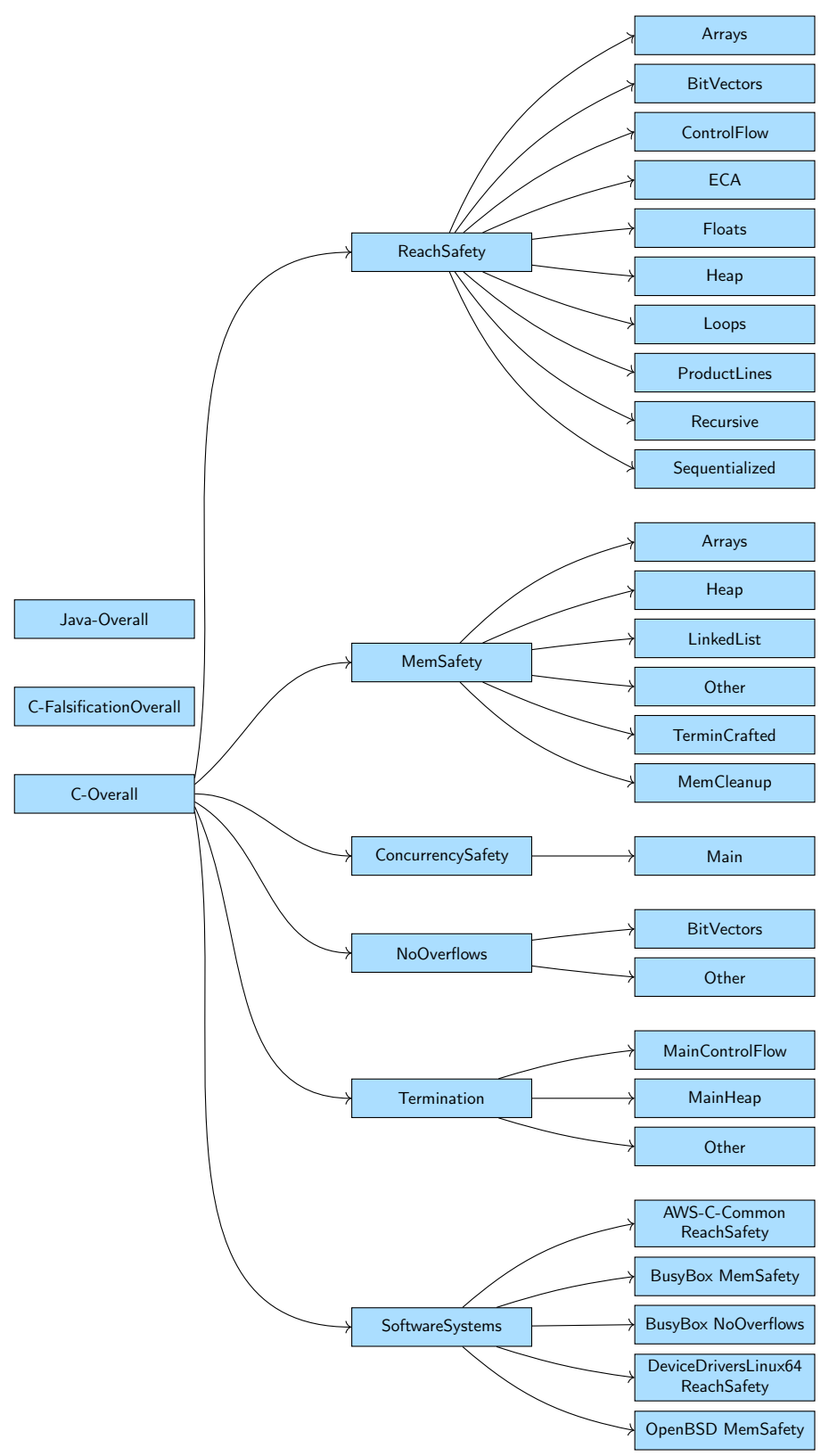

Fig. 2: Category structure for SV-COMP 2020; category C-FalsificationOverall contains all verification tasks of $C$-Overall without Termination; Java-Overall contains all Java verification tasks 
Table 1: Properties used in SV-COMP 2020 (unchanged since 2019 [14])

\begin{tabular}{|c|c|}
\hline Formula & Interpretation \\
\hline G! call (foo()) & A call to function foo is not reachable on any finite execution. \\
\hline G valid-free & $\begin{array}{l}\text { All memory deallocations are valid (counterexample: invalid free). } \\
\text { More precisely: There exists no finite execution of the program } \\
\text { during which an invalid memory deallocation occurs. }\end{array}$ \\
\hline G valid-deref & $\begin{array}{l}\text { All pointer dereferences are valid (counterexample: invalid } \\
\text { dereference). More precisely: There exists no finite execution of } \\
\text { the program during which an invalid pointer dereference occurs. }\end{array}$ \\
\hline G valid-memtrack & $\begin{array}{l}\text { All allocated memory is tracked, i.e., pointed to or deallocated } \\
\text { (counterexample: memory leak). More precisely: There exists } \\
\text { no finite execution of the program during which the program lost } \\
\text { track of some previously allocated memory. }\end{array}$ \\
\hline G valid-memcleanup & $\begin{array}{l}\text { All allocated memory is deallocated before the program } \\
\text { terminates. In addition to valid-memtrack: There exists } \\
\text { no finite execution of the program during which the program } \\
\text { terminates but still points to allocated memory. } \\
\text { (Comparison to Valgrind: This property can be violated even } \\
\text { if Valgrind reports 'still reachable'.) }\end{array}$ \\
\hline$F$ end & $\begin{array}{l}\text { All program executions are finite and end on proposition end, } \\
\text { which marks all program exits (counterexample: infinite loop). } \\
\text { More precisely: There exists no execution of the program on } \\
\text { which the program never terminates. }\end{array}$ \\
\hline
\end{tabular}

Table 2: Scoring schema for SV-COMP 2020 (unchanged since 2017 [13])

\begin{tabular}{lrl}
\hline Reported result & Points & Description \\
\hline UNKNOWN & 0 & Failure to compute verification result \\
FALSE correct & +1 & $\begin{array}{l}\text { Violation of property in program was correctly found } \\
\text { and a validator confirmed the result based on a witness }\end{array}$ \\
FALSE incorrect & -16 & $\begin{array}{l}\text { Violation reported but property holds (false alarm) } \\
\text { TRUE correct }\end{array}$ \\
& +2 & $\begin{array}{l}\text { Program correctly reported to satisfy property } \\
\text { and a validator confirmed the result based on a witness }\end{array}$ \\
TRUE correct & +1 & $\begin{array}{l}\text { Program correctly reported to satisfy property, } \\
\text { unconfirmed }\end{array}$ \\
TRUE incorrect & -32 & Incorrect program reported as correct (wrong proof) \\
\hline
\end{tabular}

repository. Table 1 lists the properties and their syntactical representation as overview. Property G valid-memcleanup, and thus, the category MemCleanup, was used for the first time in SV-COMP 2019. The categories AWS-C-Common and OpenBSD were added for SV-COMP 2020.

The scoring schema is identical for SV-COMP 2017-2020: Table 2 provides the overview and Fig. 3 visually illustrates the score assignment for one property. The scoring schema still contains the special rule for unconfirmed correct results for expected result TRUE that was introduced in the transitioning phase: one point is assigned if the answer matches the expected result but the witness was not confirmed. 


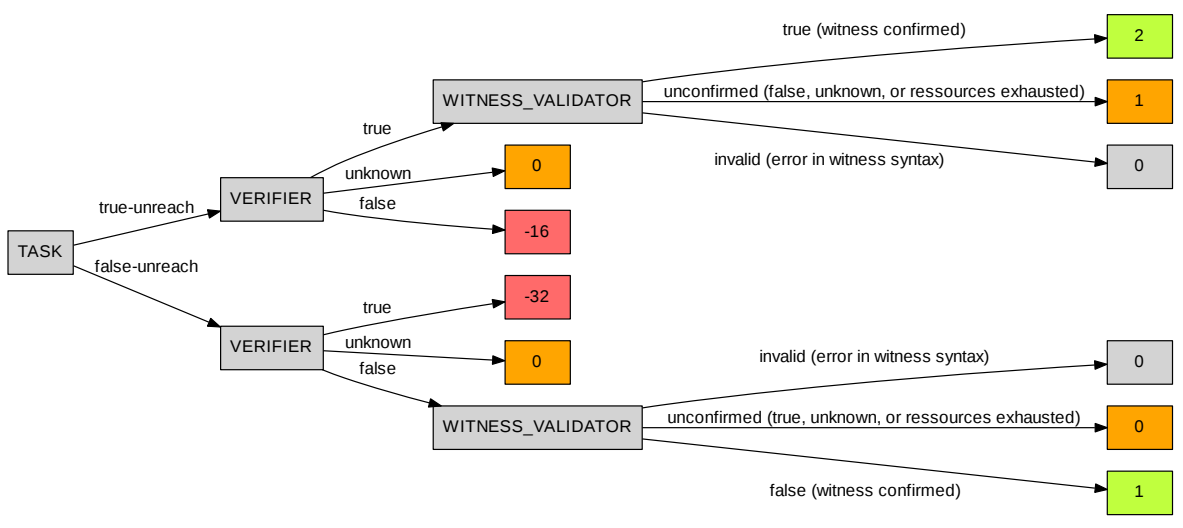

Fig. 3: Visualization of the scoring schema for the reachability property (from [13], (C) Springer-Verlag)

The ranking was again decided based on the sum of points (normalized for meta categories). In case of a tie, the ranking was decided based on success run time, which is the total CPU time over all verification tasks for which the verifier reported a correct verification result. Opt-out from Categories and Score Normalization for Meta Categories was done as described previously [9] (page 597).

\section{Reproducibility}

All major components used in the competition are available in public version repositories. This allows independent replication of the SV-COMP experiments. An overview of the components that contribute to the reproducible setup of SVCOMP is provided in Fig. 4, and the details are given in Table 3. The SV-COMP 2016 report [12] describes all components of the SV-COMP organization and how we ensure that all parts are publicly available for maximal replicability.

We have published the competition artifacts at Zenodo to guarantee their long-term availability and immutability. These artifacts comprise the verification tasks, the produced competition results, and the produced verification witnesses. The DOIs and references are given in Table 4. The archive for the competition results includes the raw results in BENCHEXEC's XML exchange format, the log output of the verifiers and validators, and a mapping from files names to SHA-256 hashes. The hashes of the files are useful for validating the exact contents of a file, and accessing the files inside the archive that contains the verification witnesses.

To provide a more transparent way of accessing the exact versions of the verifiers that were used in the competition, all verifier archives are stored in a public Git repository. GiTLAB was used to host the repository for the verifier archives due to its generous repository size limit of $10 \mathrm{~GB}$. The final size of the Git repository is $5.78 \mathrm{~GB}$. 


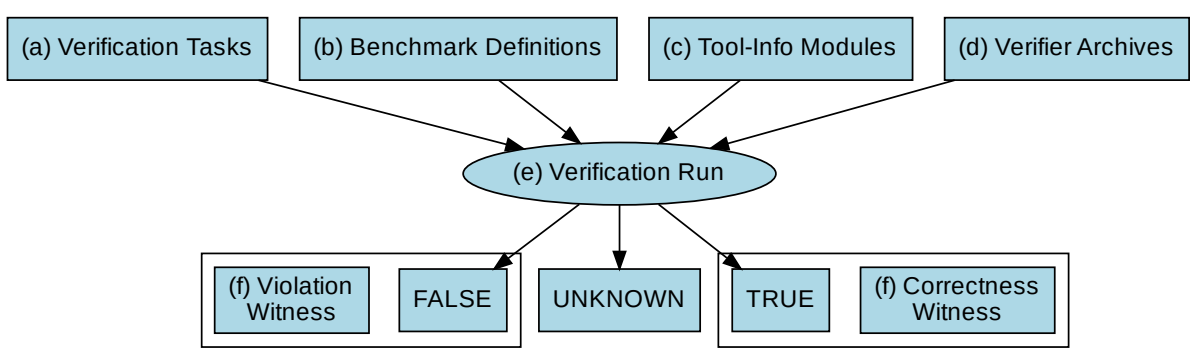

Fig. 4: SV-COMP components and the execution flow

Table 3: Publicly available components for replicating SV-COMP 2020

\begin{tabular}{llll}
\hline Component & Fig. 4 & Repository & Version \\
\hline Verification Tasks & (a) & github.com/sosy-lab/sv-benchmarks & svcomp20 \\
Benchmark Definitions & (b) & github.com/sosy-lab/sv-comp & svcomp20 \\
Tool-Info Modules & (c) & github.com/sosy-lab/benchexec & 2.5 .1 \\
Verifier Archives & (d) & gitlab.com/sosy-lab/sv-comp/archives-2020 & svcomp20 \\
Benchmarking & (e) & github.com/sosy-lab/benchexec & 2.5 .1 \\
Witness Format & (f) & github.com/sosy-lab/sv-witnesses & svcomp20 \\
\hline
\end{tabular}

\section{Results and Discussion}

The results of the competition experiments represent the state of the art in fully automatic software-verification tools. The report shows the results, in terms of effectiveness (number of verification tasks that can be solved and correctness of the results, as accumulated in the score) and efficiency (resource consumption in terms of CPU time). The results are presented in the same way as in last years, such that the improvements compared to last year are easy to identify. The results presented in this report were inspected and approved by the participating teams. We now discuss the highlights of the results.

Participating Verifiers. Table 5 and the competition web site ${ }^{14}$ provide an overview of the participating verification systems. Table 6 lists the algorithms and techniques that are used in the verification tools.

Computing Resources. The resource limits were the same as in the previous competitions [12]: Each verification run was limited to 8 processing units (cores), $15 \mathrm{~GB}$ of memory, and $15 \mathrm{~min}$ of $\mathrm{CPU}$ time. The witness validation was limited to 2 processing units, $7 \mathrm{~GB}$ of memory, and $1.5 \mathrm{~min}$ of $\mathrm{CPU}$ time for violation witnesses and $15 \mathrm{~min}$ of $\mathrm{CPU}$ time for correctness witnesses. The machines for running the experiments are part of a compute cluster that consists of

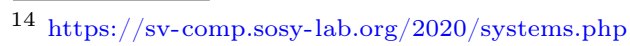


Table 4: Artifacts published for SV-COMP 2020

\begin{tabular}{llc}
\hline Content & DOI & Reference \\
\hline Verification Tasks & $10.5281 /$ zenodo.3633334 & {$[17]$} \\
Competition Results & $10.5281 /$ zenodo.3630205 & {$[16]$} \\
Verification Witnesses & $10.5281 /$ zenodo.3630188 & {$[18]$} \\
\hline
\end{tabular}

Table 5: Competition candidates with tool references and representing jury members

\begin{tabular}{|c|c|c|c|}
\hline Participant & Ref. & Jury member & Affiliation \\
\hline $2 \mathrm{LS}$ & {$[26,55]$} & Viktor Malík & BUT, Brno, Czechia \\
\hline BRICK & & Lei $\mathrm{Bu}$ & Nanjing U., China \\
\hline Свмс & [51] & Michael Tautschnig & Amazon Web Services, UK \\
\hline Coastal & {$[67]$} & Willem Visser & Stellenbosch U., South Africa \\
\hline CPA-BAM-BNB & {$[3,68]$} & Vadim Mutilin & ISP RAS, Russia \\
\hline CPA-LOCKATOR & {$[4]$} & Pavel Andrianov & ISP RAS, Russia \\
\hline CPA-SEQ & {$[22,36]$} & Martin Spiessl & LMU Munich, Germany \\
\hline DARTAGNAN & {$[40,53]$} & Hernán Ponce de León & Bundeswehr U. Munich, Germany \\
\hline Divine & {$[6,52]$} & Henrich Lauko & Masaryk U., Czechia \\
\hline EsBmC & {$[38,39]$} & Felipe R. Monteiro & Federal U. of Amazonas, Brazil \\
\hline GACAL & [61] & Benjamin Quiring & Northeastern U., USA \\
\hline JAVA-RANGER & [65] & Vaibhav Sharma & U. of Minnesota, USA \\
\hline JAYHORN & {$[49,50]$} & Philipp Ruemmer & Uppsala U., Sweden \\
\hline JBмC & {$[33,34]$} & Peter Schrammel & U. of Sussex, UK \\
\hline JDART & {$[54,56]$} & Falk Howar & TU Dortmund, Germany \\
\hline LAZY-CSEQ & {$[47,48]$} & Omar Inverso & Gran Sasso Science Inst., Italy \\
\hline МAP2CHECK & {$[63,64]$} & Herbert Rocha & Federal U. of Roraima, Brazil \\
\hline PeSCo & {$[35,62]$} & Cedric Richter & Paderborn U., Germany \\
\hline Pinaka & [30] & Saurabh Joshi & IIT Hyderabad, India \\
\hline PredatorhP & {$[44,59]$} & Veronika Šoková & BUT, Brno, Czechia \\
\hline $\mathrm{SPF}$ & {$[57,60]$} & Willem Visser & Amazon, USA \\
\hline Symвiотіс & {$[28,29]$} & Marek Chalupa & Masaryk U., Czechia \\
\hline UAutomizer & {$[42,43]$} & Matthias Heizmann & U. of Freiburg, Germany \\
\hline UKOJAK & {$[27,58]$} & Alexander Nutz & U. of Freiburg, Germany \\
\hline UTAIPAN & {$[37,41]$} & Daniel Dietsch & U. of Freiburg, Germany \\
\hline VERIAbs & {$[1,2]$} & Priyanka Darke & Tata Consultancy Services, India \\
\hline VERIFuzz & [31] & Raveendra Kumar M. & Tata Consultancy Services, India \\
\hline YogAR-CBMC & {$[70,71]$} & Liangze Yin & Nat. U. of Defense Techn., China \\
\hline
\end{tabular}

168 machines; each verification run was executed on an otherwise completely unloaded, dedicated machine, in order to achieve precise measurements. Each machine had one Intel Xeon E3-1230 v5 CPU, with 8 processing units each, a frequency of $3.4 \mathrm{GHz}, 33 \mathrm{~GB}$ of RAM, and a GNU/Linux operating system (x86_64-linux, Ubuntu 18.04 with Linux kernel 4.15). We used BenchExec [23] to measure and control computing resources (CPU time, memory, CPU energy) and VERIFIERCLOUD ${ }^{15}$ to distribute, install, run, and clean-up verification runs,

15 https://vcloud.sosy-lab.org 
Table 6: Algorithms and techniques that the competition candidates offer

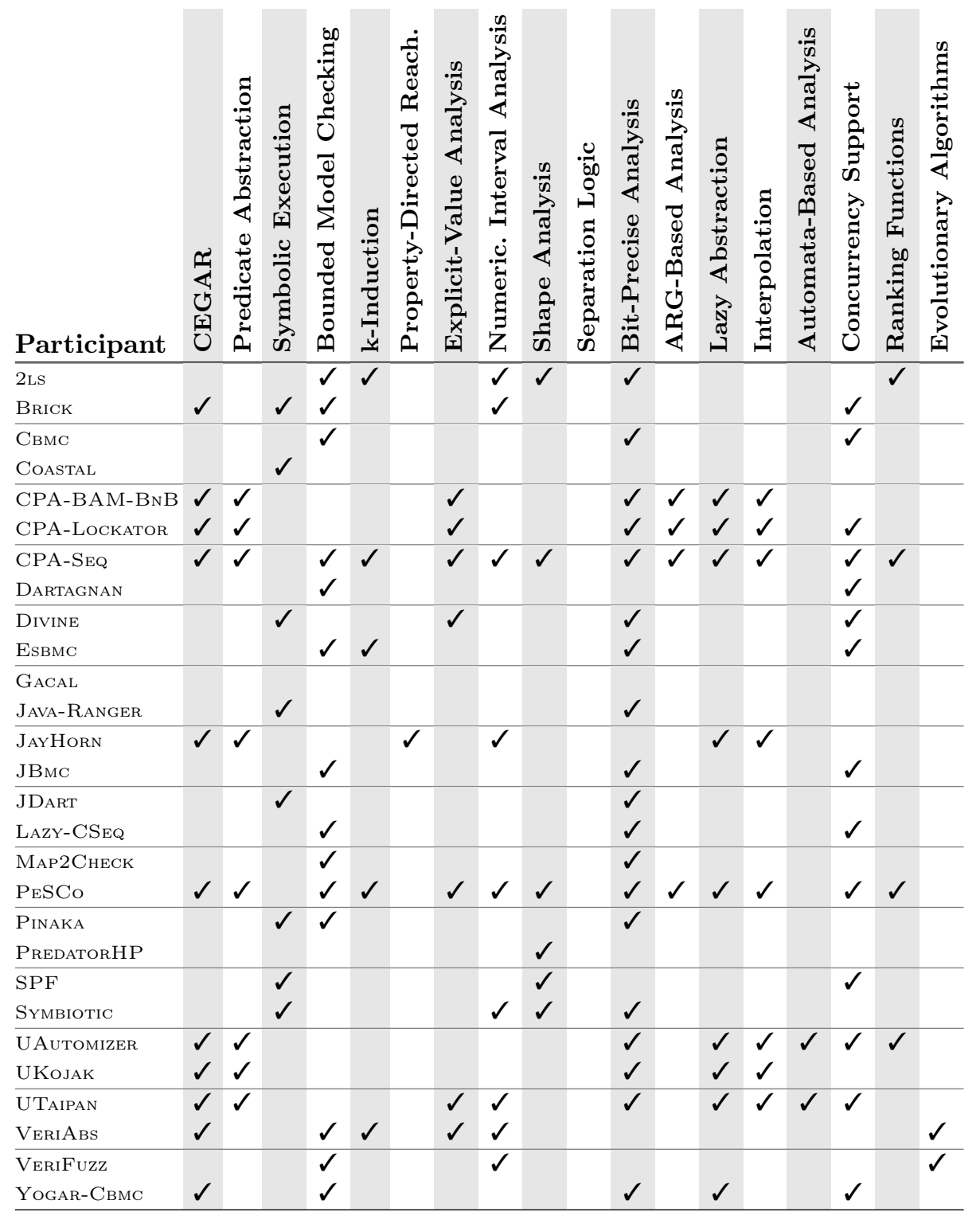


and to collect the results. The values for time and energy are accumulated over all cores of the CPU. To measure the CPU energy, we use CPU EnERGY Meter [24] (integrated in BenchExec [23]).

One complete verification execution of the competition consisted of 138074 verification runs (each verifier on each verification task of the selected categories according to the opt-outs), consuming 491 days of CPU time and $130 \mathrm{kWh}$ of CPU energy (without validation). Witness-based result validation required 684858 validation runs (each validator on each verification task for categories with witness validation, and for each verifier), consuming 311 days of CPU time. Each tool was executed several times, in order to make sure no installation issues occur during the execution. Including preruns, the infrastructure managed a total of 1018781 verification runs consuming 4.8 years of $\mathrm{CPU}$ time, and 10705227 validation runs consuming 6.9 years of CPU time.

Quantitative Results. Table 7 presents the quantitative overview of all tools and all categories. The head row mentions the category, the maximal score for the category, and the number of verification tasks. The tools are listed in alphabetical order; every table row lists the scores of one verifier. We indicate the top three candidates by formatting their scores in bold face and in larger font size. An empty table cell means that the verifier opted-out from the respective main category (perhaps participating in subcategories only, restricting the evaluation to a specific topic). More information (including interactive tables, quantile plots for every category, and also the raw data in XML format) is available on the competition web site ${ }^{16}$ and in the results artifact (see Table 4).

Table 8 reports the top three verifiers for each category. The run time (column 'CPU Time') and energy (column 'CPU Energy') refer to successfully solved verification tasks (column 'Solved Tasks'). We also report the number of tasks for which no witness validator was able to confirm the result (column 'Unconf. Tasks'). The columns 'False Alarms' and 'Wrong Proofs' report the number of verification tasks for which the verifier reported wrong results, i.e., reporting a counterexample when the property holds (incorrect FALSE) and claiming that the program fulfills the property although it actually contains a bug (incorrect TRUE), respectively.

Score-Based Quantile Functions for Quality Assessment. We use scorebased quantile functions $[9,23]$ because these visualizations make it easier to understand the results of the comparative evaluation. The web site ${ }^{16}$ and the results archive (see Table 4) include such a plot for each category. As an example, we show the plot for category $C$-Overall (all verification tasks) in Fig. 5. A total of 11 verifiers participated in category $C$-Overall, for which the quantile plot shows the overall performance over all categories (scores for meta categories are normalized [9]). A more detailed discussion of score-based quantile plots, including examples of what insights one can obtain from the plots, is provided in previous competition reports $[9,12]$.

16 https://sv-comp.sosy-lab.org/2020/results 
Table 7: Quantitative overview over all results; empty cells represent opt-outs

\begin{tabular}{|c|c|c|c|c|c|c|c|c|c|}
\hline Participant & 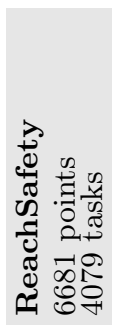 & 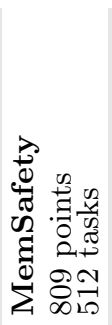 & 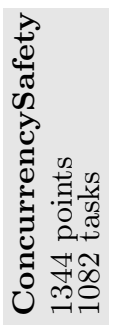 & 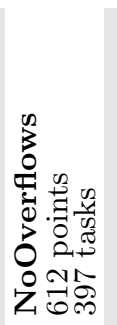 & 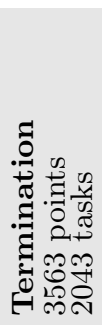 & 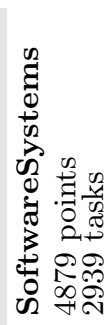 & 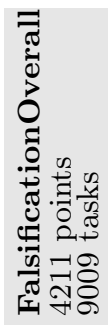 & 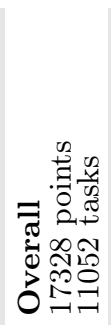 & 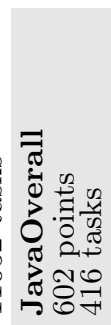 \\
\hline $2 \mathrm{LS}$ & 2491 & 298 & 0 & 340 & 1264 & 13 & 914 & 4924 & \\
\hline \multicolumn{10}{|l|}{ Brick } \\
\hline Свмс & 2864 & -162 & 554 & 268 & 499 & 30 & 1256 & 3365 & \\
\hline CPA-BAM-BNB & & & & & & 602 & & & \\
\hline CPA-SEQ & 4396 & 355 & 996 & 483 & 1720 & 746 & 2772 & 9219 & \\
\hline CPA-LOCKATOR & & & -387 & & & & & & \\
\hline DARTAGNAN & & & 173 & & & & & & \\
\hline Divine & -76 & 71 & 550 & 0 & 0 & -12 & 585 & 1151 & \\
\hline EsBMC & 3481 & 334 & 325 & 264 & 777 & 500 & 1639 & 5567 & \\
\hline \multicolumn{10}{|l|}{ GACAL } \\
\hline LAZY-CSEQ & & & 1279 & & & & & & \\
\hline MAP2CHECK & & -68 & & -89 & & & & & \\
\hline PESCo & 4376 & & & & & & 1590 & 8023 & \\
\hline Pinaka & 2585 & & & 243 & 590 & & & & \\
\hline PredatorhP & & 611 & & & & & & & \\
\hline Symbiotic & 2753 & 516 & 0 & 294 & 1022 & 954 & 1828 & 5985 & \\
\hline UAUTOMIZER & 2696 & 354 & 296 & 466 & 2942 & 591 & 893 & 8178 & \\
\hline UKOJAK & 1702 & 231 & 0 & 387 & 0 & 501 & 1148 & 3710 & \\
\hline UTAIPAN & 2185 & 316 & 289 & 461 & 0 & 482 & 805 & 5057 & \\
\hline VeriAbs & 5543 & 0 & 0 & 0 & 0 & 244 & 273 & 2656 & \\
\hline VeriFuzz & 1206 & & & 146 & & & & & \\
\hline YogAR-CBMC & & & 1275 & & & & & & \\
\hline Coastal & & & & & & & & & 472 \\
\hline J AVA-RANGER & & & & & & & & & 549 \\
\hline J AYHORN & & & & & & & & & 278 \\
\hline JBMC & & & & & & & & & 527 \\
\hline JDART & & & & & & & & & 524 \\
\hline SPF & & & & & & & & & 410 \\
\hline
\end{tabular}


Table 8: Overview of the top-three verifiers for each category (measurement values for CPU time and energy rounded to two significant digits)

\begin{tabular}{|c|c|c|c|c|c|c|c|c|}
\hline Rank & Verifier & Score & $\begin{array}{l}\text { CPU } \\
\text { Time } \\
(\text { in h })\end{array}$ & $\begin{array}{c}\text { CPU } \\
\text { Energy } \\
\text { (in kWh) }\end{array}$ & $\begin{array}{c}\text { Solved } \\
\text { Tasks }\end{array}$ & $\begin{array}{c}\text { Unconf. } \\
\text { Tasks }\end{array}$ & $\begin{array}{c}\text { False } \\
\text { Alarms }\end{array}$ & $\begin{array}{l}\text { Wrong } \\
\text { Proofs }\end{array}$ \\
\hline \multicolumn{9}{|c|}{ ReachSafety } \\
\hline 1 & VeriAbs & 5543 & 150 & 1.6 & 3412 & 171 & & \\
\hline 2 & CPA-SEQ & 4396 & 72 & .75 & 2700 & 54 & 8 & \\
\hline 3 & PeSCo & 4376 & 39 & .38 & 2518 & 36 & 4 & \\
\hline \multicolumn{9}{|c|}{ MemSafety } \\
\hline 1 & PREDATORHP & 611 & .78 & .010 & 392 & 15 & & \\
\hline 2 & Symbiotic & 516 & .51 & .010 & 358 & 6 & & \\
\hline 3 & CPA-SEQ & 355 & .76 & .010 & 264 & 1 & & \\
\hline \multicolumn{9}{|c|}{ ConcurrencySafety } \\
\hline 1 & LAZY-CSEQ & 1279 & 6.7 & .090 & 1023 & 44 & & \\
\hline 2 & YogAR-CBMC & 1275 & .39 & .000 & 1024 & 33 & & \\
\hline 3 & CPA-SEQ & 996 & 12 & .11 & 830 & 102 & & \\
\hline \multicolumn{9}{|c|}{ NoOverflows } \\
\hline 1 & CPA-SEQ & 483 & .93 & .010 & 321 & 8 & & \\
\hline 2 & UAUTOMIZER & 466 & 1.4 & .010 & 326 & 0 & & \\
\hline 3 & UTAIPAN & 461 & 1.5 & .010 & 323 & 0 & & \\
\hline \multicolumn{9}{|c|}{ Termination } \\
\hline 1 & UAUTOMIZER & 2942 & 15 & .16 & 1606 & 7 & & \\
\hline 2 & CPA-SEQ & 1720 & 16 & .17 & 1247 & 7 & & \\
\hline 3 & $2 \mathrm{LS}$ & 1264 & 3.2 & .030 & 955 & 361 & 3 & \\
\hline \multicolumn{9}{|c|}{ SoftwareSystems } \\
\hline 1 & SYMBIOTIC & 954 & .25 & .000 & 676 & 36 & 3 & 1 \\
\hline 2 & CPA-SEQ & 746 & 21 & .24 & 1381 & 363 & 1 & \\
\hline 3 & CPA-BAM-BNB & 602 & 8.0 & .070 & 1411 & 582 & 3 & 4 \\
\hline \multicolumn{9}{|c|}{ FalsificationOverall } \\
\hline 1 & CPA-SEQ & 2772 & 45 & .45 & 2240 & 139 & 9 & \\
\hline 2 & Symbiotic & 1828 & 27 & .35 & 1461 & 10 & 3 & \\
\hline 3 & EsBmC & 1639 & 14 & .18 & 1819 & 385 & 16 & \\
\hline \multicolumn{9}{|c|}{ Overall } \\
\hline 1 & CPA-SEQ & 9219 & 120 & 1.3 & 6743 & 535 & 9 & \\
\hline 2 & UAUTOMIZER & 8178 & 83 & .84 & 5523 & 693 & 71 & 2 \\
\hline 3 & PESCo & 8023 & 120 & 1.2 & 6402 & 242 & 32 & \\
\hline \multicolumn{9}{|c|}{ JavaOverall } \\
\hline 1 & J AVA-RANGER & 549 & 1.3 & .010 & 376 & & & \\
\hline 2 & $\mathrm{JBMC}$ & 527 & .18 & .000 & 376 & & & \\
\hline 3 & JDART & 524 & .26 & .000 & 374 & & & \\
\hline
\end{tabular}




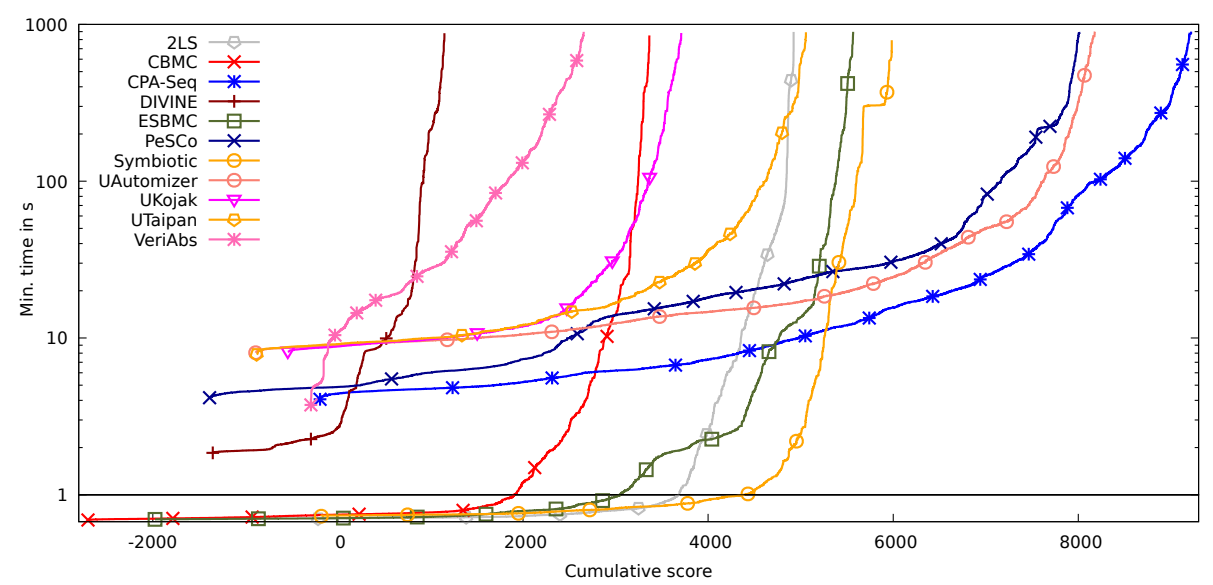

Fig. 5: Quantile functions for category $C$-Overall. Each quantile function illustrates the quantile ( $x$-coordinate) of the scores obtained by correct verification runs below a certain run time ( $y$-coordinate). More details were given previously [9]. A logarithmic scale is used for the time range from $1 \mathrm{~s}$ to $1000 \mathrm{~s}$, and a linear scale is used for the time range between $0 \mathrm{~s}$ and $1 \mathrm{~s}$.

Alternative Rankings. The community suggested to report a couple of alternative rankings that honor different aspects of the verification process as complement to the official SV-COMP ranking. Table 9 is similar to Table 8 , but contains the alternative ranking categories Correct and Green Verifiers. Column 'Quality' gives the score in score points, column 'CPU Time' the CPU usage of successful runs in hours, column 'CPU Energy' the CPU usage of successful runs in kWh, column 'Solved Tasks' the number of correct results, column 'Wrong Results' the sum of false alarms and wrong proofs in number of errors, and column 'Rank Measure' gives the measure to determine the alternative rank.

Correct Verifiers - Low Failure Rate. The right-most columns of Table 8 report that the verifiers achieve a high degree of correctness (all top three verifiers in the $\mathrm{C}$ track have less than $2 \%$ wrong results). The winners of category Java-Overall produced not a single wrong answer. The first category in Table 9 uses a failure rate as

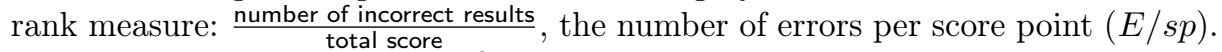
We use $E$ as unit for number of incorrect results and $s p$ as unit for total score. It is remarkable to see that the worst result was $0.38 \mathrm{E} / \mathrm{sp}$ in SV-COMP 2019 and is now improved to $0.032 \mathrm{E} / \mathrm{sp}$, with is an order of magnitude better.

Green Verifiers - Low Energy Consumption. Since a large part of the cost of verification is given by the energy consumption, it might be important to also consider the energy efficiency. The second category in Table 9 uses the energy consumption per score point as rank measure: $\frac{\text { total CPU energy }}{\text { total score }}$, with the unit $J / s p$. It is interesting to see that the worst result from SV-COMP 2019 was $4200 \mathrm{~J} / \mathrm{sp}$, and now it is improved to $2200 \mathrm{~J} / \mathrm{sp}$. 
Table 9: Alternative rankings; quality is given in score points (sp), CPU time in hours (h), energy in kilojoule (kJ), wrong results in errors (E), rank measures in errors per score point $(\mathrm{E} / \mathrm{sp})$, joule per score point $(\mathrm{J} / \mathrm{sp})$, and score points $(\mathrm{sp})$

\begin{tabular}{lccccccc}
\hline Rank & Verifier & Quality & $\begin{array}{c}\text { CPU } \\
\text { Time } \\
(\mathbf{h})\end{array}$ & $\begin{array}{c}\text { CPU } \\
\text { Energy } \\
(\mathbf{k W h})\end{array}$ & $\begin{array}{c}\text { Solved } \\
\text { Tasks }\end{array}$ & $\begin{array}{c}\text { Wrong } \\
\text { Results } \\
(\mathbf{E})\end{array}$ & $\begin{array}{c}\text { Rank } \\
\text { Measure }\end{array}$ \\
\hline Correct & Verifiers & & & & & & $(\mathrm{E} / \mathrm{sp})$ \\
1 & CPA-SEQ & 9219 & 120 & 1.3 & 6743 & 9 & .0010 \\
2 & UKoJAK & 3710 & 48 & 0.49 & 2405 & 4 & .0011 \\
3 & 2LS & 4924 & 27 & 0.24 & 3044 & 8 & .0016 \\
worst & & & & & & & .032 \\
\hline Green & Verifiers & & & & & & $(\mathrm{J} / \mathrm{sp})$ \\
1 & CвMC & 3365 & 15 & 0.16 & 3217 & 67 & 170 \\
2 & 2LS & 4924 & 27 & 0.24 & 3044 & 8 & 180 \\
3 & EsBMC & 5567 & 35 & 0.41 & 5520 & 51 & 270 \\
worst & & & & & & & 2200 \\
\hline
\end{tabular}

Table 10: Confirmation rate of verification witnesses in SV-COMP 2020

\begin{tabular}{|c|c|c|c|c|c|c|c|c|}
\hline \multirow{3}{*}{$\begin{array}{l}\text { Result } \\
2 \mathrm{LS}\end{array}$} & \multicolumn{4}{|c|}{ True } & \multicolumn{4}{|c|}{ FALSE } \\
\hline & \multirow{2}{*}{$\frac{\text { Total }}{2060}$} & \multicolumn{2}{|c|}{ Confirmed } & \multirow{2}{*}{$\frac{\text { Unconf. }}{11}$} & \multirow{2}{*}{$\frac{\text { Total }}{1449}$} & \multicolumn{2}{|c|}{ Confirmed } & \multirow{2}{*}{$\frac{\text { Unconf. }}{454}$} \\
\hline & & 2049 & $99 \%$ & & & 995 & $69 \%$ & \\
\hline CвMC & 1949 & 1821 & $93 \%$ & 128 & 2095 & 1396 & $67 \%$ & 699 \\
\hline CPA-SEQ & 4347 & 3958 & $91 \%$ & 389 & 2931 & 2785 & $95 \%$ & 146 \\
\hline Divine & 811 & 793 & $98 \%$ & 18 & 1099 & 672 & $61 \%$ & 427 \\
\hline EsBmc & 3779 & 3701 & $98 \%$ & 78 & 2204 & 1819 & $83 \%$ & 385 \\
\hline PESCO & 3777 & 3704 & $98 \%$ & 73 & 2867 & 2698 & $94 \%$ & 169 \\
\hline Symbiotic & 2196 & 2146 & $98 \%$ & 50 & 1996 & 1879 & $94 \%$ & 117 \\
\hline UAutomizer & 4135 & 4029 & $97 \%$ & 106 & 2081 & 1494 & $72 \%$ & 587 \\
\hline UKOJAK & 1811 & 1801 & $99 \%$ & 10 & 606 & 604 & $100 \%$ & 2 \\
\hline UTAIPAN & 2496 & 2438 & $98 \%$ & 58 & 1308 & 730 & $56 \%$ & 578 \\
\hline VERIABS & 3908 & 3387 & $87 \%$ & 521 & 1536 & 1332 & $87 \%$ & 204 \\
\hline
\end{tabular}

Verifiable Witnesses. All SV-COMP verifiers are required to justify the result (TRUE or FALSE) by producing a verification witness (except for those categories for which no witness validator is available). We used six independently developed witness-based result validators [19, 20, 21, 25, 66].

The majority of witnesses that the verifiers produced can be confirmed by the results-validation process. Interestingly, the confirmation rate for the TRUE results is significantly higher than for the FALSE results. Table 10 shows the confirmed versus unconfirmed results: the first column lists the verifiers 


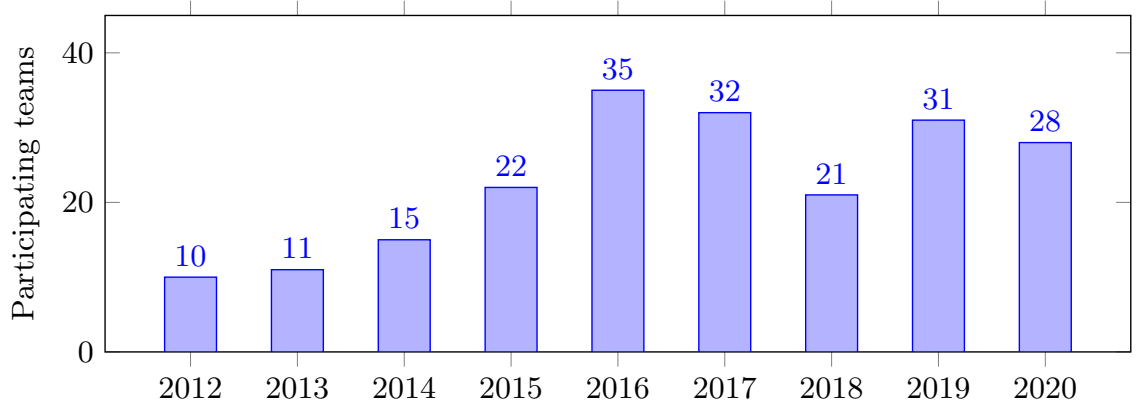

Fig. 6: Number of participating teams for each year

of category $C$-Overall, the three columns for result TRUE reports the total, confirmed, and unconfirmed number of verification tasks for which the verifier answered with TRUE, respectively, and the three columns for result FALSE reports the total, confirmed, and unconfirmed number of verification tasks for which the verifier answered with FALSE, respectively. More information (for all verifiers) is given in the detailed tables on the competition web site ${ }^{16}$ and in the results artifact; all verification witnesses are also contained in the witnesses artifact (see Table 4). Result validation is an important topic also in other competitions (e.g., in the SAT competition [5, 69]).

\section{Conclusion}

SV-COMP 2020, the $9^{\text {th }}$ edition of the Competition on Software Verification, attracted 28 participating teams from 11 countries (see Fig. 6 for the participation numbers). SV-COMP continues to offer a broad overview of the state of the art in automatic software verification. The competition does not only execute the verifiers and collect results, but also validates the verification results, using six independently developed results validators. The number of verification tasks was increased to 11052 in $\mathrm{C}$ and to 416 in Java. As before, the large jury and the organizer made sure that the competition follows the high quality standards of the TACAS conference, in particular with respect to the important principles of fairness, community support, and transparency.

Data Availability Statement. The verification tasks and results of the competition are published at Zenodo, as described in Table 4. All components and data that are necessary for reproducing the competition are available in public version repositories, as specified in Fig. 4 and Table 3. Furthermore, the results are presented online on the competition web site for easy access: https://sv-comp.sosy-lab.org/2020/results/. 


\section{References}

1. Afzal, M., Asia, A., Chauhan, A., Chimdyalwar, B., Darke, P., Datar, A., Kumar, S., Venkatesh, R.: VeriABs: Verification by abstraction and test generation. In: Proc. ASE. pp. 1138-1141 (2019). https://doi.org/10.1109/ASE.2019.00121

2. Afzal, M., Chakraborty, S., Chauhan, A., Chimdyalwar, B., Darke, P., Gupta, A., Kumar, S., M., C.B., Unadkat, D., Venkatesh, R.: VeriAbs: Verification by abstraction and test generation (competition contribution). In: Proc. TACAS (2). LNCS 12079, Springer (2020)

3. Andrianov, P., Friedberger, K., Mandrykin, M.U., Mutilin, V.S., Volkov, A.: CPABAM-BNB: Block-abstraction memoization and region-based memory models for predicate abstractions (competition contribution). In: Proc. TACAS. pp. 355-359. LNCS 10206, Springer (2017). https://doi.org/10.1007/978-3-662-54580-5_22

4. Andrianov, P., Mutilin, V., Khoroshilov, A.: Predicate abstraction based configurable method for data race detection in Linux kernel. In: Proc. TMPA. CCIS 779, Springer (2018). https://doi.org/10.1007/978-3-319-71734-0_2

5. Balyo, T., Heule, M.J.H., Järvisalo, M.: SAT Competition 2016: Recent developments. In: Proc. AAAI. pp. 5061-5063. AAAI Press (2017)

6. Baranová, Z., Barnat, J., Kejstová, K., Kučera, T., Lauko, H., Mrázek, J., Ročkai, P., Štill, V.: Model checking of C and C++ with Divine 4. In: Proc. ATVA. pp. 201-207. LNCS 10482, Springer (2017). https://doi.org/10.1007/978-3-319-68167-2_14

7. Bartocci, E., Beyer, D., Black, P.E., Fedyukovich, G., Garavel, H., Hartmanns, A., Huisman, M., Kordon, F., Nagele, J., Sighireanu, M., Steffen, B., Suda, M., Sutcliffe, G., Weber, T., Yamada, A.: TOOLympics 2019: An overview of competitions in formal methods. In: Proc. TACAS (3). pp. 3-24. LNCS 11429, Springer (2019). https://doi.org/10.1007/978-3-030-17502-3_1

8. Beyer, D.: Competition on software verification (SV-COMP). In: Proc. TACAS. pp. 504-524. LNCS 7214, Springer (2012). https://doi.org/10.1007/978-3-642-28756$5 \_38$

9. Beyer, D.: Second competition on software verification (Summary of SVCOMP 2013). In: Proc. TACAS. pp. 594-609. LNCS 7795, Springer (2013). https://doi.org/10.1007/978-3-642-36742-7_43

10. Beyer, D.: Status report on software verification (Competition summary SVCOMP 2014). In: Proc. TACAS. pp. 373-388. LNCS 8413, Springer (2014). https://doi.org/10.1007/978-3-642-54862-8_25

11. Beyer, D.: Software verification and verifiable witnesses (Report on SVCOMP 2015). In: Proc. TACAS. pp. 401-416. LNCS 9035, Springer (2015). https://doi.org/10.1007/978-3-662-46681-0_31

12. Beyer, D.: Reliable and reproducible competition results with BenchExEC and witnesses (Report on SV-COMP 2016). In: Proc. TACAS. pp. 887-904. LNCS 9636, Springer (2016). https://doi.org/10.1007/978-3-662-49674-9_55

13. Beyer, D.: Software verification with validation of results (Report on SVCOMP 2017). In: Proc. TACAS. pp. 331-349. LNCS 10206, Springer (2017). https://doi.org/10.1007/978-3-662-54580-5_20

14. Beyer, D.: Automatic verification of $\bar{C}$ and Java programs: SV-COMP 2019. In: Proc. TACAS (3). pp. 133-155. LNCS 11429, Springer (2019). https://doi.org/10.1007/978-3-030-17502-3_9

15. Beyer, D.: First international competition on software testing (Test-Comp 2019). Int. J. Softw. Tools Technol. Transf. (2020) 
16. Beyer, D.: Results of the 9th International Competition on Software Verification (SV-COMP 2020). Zenodo (2020). https://doi.org/10.5281/zenodo.3630205

17. Beyer, D.: SV-Benchmarks: Benchmark set of 9th Intl. Competition on Software Verification (SV-COMP 2020). Zenodo (2020). https://doi.org/10.5281/zenodo.3633334

18. Beyer, D.: Verification witnesses from SV-COMP 2020 verification tools. Zenodo (2020). https://doi.org/10.5281/zenodo.3630188

19. Beyer, D., Dangl, M., Dietsch, D., Heizmann, M.: Correctness witnesses: Exchanging verification results between verifiers. In: Proc. FSE. pp. 326-337. ACM (2016). https://doi.org/10.1145/2950290.2950351

20. Beyer, D., Dangl, M., Dietsch, D., Heizmann, M., Stahlbauer, A.: Witness validation and stepwise testification across software verifiers. In: Proc. FSE. pp. 721-733. ACM (2015). https://doi.org/10.1145/2786805.2786867

21. Beyer, D., Dangl, M., Lemberger, T., Tautschnig, M.: Tests from witnesses: Execution-based validation of verification results. In: Proc. TAP. pp. 3-23. LNCS 10889, Springer (2018). https://doi.org/10.1007/978-3-319-92994-1_1

22. Beyer, D., Keremoglu, M.E.: CPACHECKER: A tool for configurable software verification. In: Proc. CAV. pp. 184-190. LNCS 6806, Springer (2011). https://doi.org/10.1007/978-3-642-22110-1_16

23. Beyer, D., Löwe, S., Wendler, P.: Reliable benchmarking: Requirements and solutions. Int. J. Softw. Tools Technol. Transfer 21(1), 1-29 (2019). https://doi.org/10.1007/s10009-017-0469-y

24. Beyer, D., Wendler, P.: CPU Energy Meter: A tool for energy-aware algorithms engineering. In: Proc. TACAS (2). LNCS 12079, Springer (2020)

25. Beyer, D., Spiessl, M.: Meta Val: Witness validation via verification. In: unpublished manuscript (2020)

26. Brain, M., Joshi, S., Kröning, D., Schrammel, P.: Safety verification and refutation by k-invariants and k-induction. In: Proc. SAS. pp. 145-161. LNCS 9291, Springer (2015). https://doi.org/10.1007/978-3-662-48288-9_9

27. Brückner, I., Dräger, K., Finkbeiner, B., Wehrheim, $\bar{H} .:$ Slicing abstractions. Fundam. Inform. 89(4), 369-392 (2008)

28. Chalupa, M., Jašek, T., Tomovič, L., Hruška, M., Šoková, V., Ayaziová, P., Strejček, J., Vojnar, T.: Symbiotic 7: Integration of Predator and more (competition contribution). In: Proc. TACAS (2). LNCS 12079, Springer (2020)

29. Chalupa, M., Strejcek, J., Vitovská, M.: Joint forces for memory safety checking. In: Proc. SPIN. pp. 115-132. Springer (2018). https://doi.org/10.1007/978-3-31994111-0_7

30. Chaudhāry, E., Joshi, S.: PinAKa: Symbolic execution meets incremental solving (competition contribution). In: Proc. TACAS (3). pp. 234-238. LNCS 11429, Springer (2019). https://doi.org/10.1007/978-3-030-17502-3_20

31. Chowdhury, A.B., Medicherla, R.K., Venkatesh, R.: VeriFuzz: Program-aware fuzzing (competition contribution). In: Proc. TACAS (3). pp. 244-249. LNCS 11429, Springer (2019). https://doi.org/10.1007/978-3-030-17502-3_22

32. Cok, D.R., Déharbe, D., Weber, T.: The 2014 SMT competition. JSAT 9, 207-242 (2016)

33. Cordeiro, L.C., Kesseli, P., Kröning, D., Schrammel, P., Trtík, M.: JBMC: A bounded model checking tool for verifying Java bytecode. In: Proc. CAV. pp. 183190. LNCS 10981, Springer (2018). https://doi.org/10.1007/978-3-319-96145-3_10

34. Cordeiro, L.C., Kröning, D., Schrammel, P.: JBmC: Bounded model checking for Java bytecode (competition contribution). In: Proc. TACAS (3). pp. 219-223. LNCS 11429, Springer (2019). https://doi.org/10.1007/978-3-030-17502-3_17 
35. Czech, M., Hüllermeier, E., Jakobs, M.C., Wehrheim, H.: Predicting rankings of software verification tools. In: Proc. SWAN. pp. 23-26. ACM (2017). https://doi.org/10.1145/3121257.3121262

36. Dangl, M., Löwe, S., Wendler, P.: CPACHECKER with support for recursive programs and floating-point arithmetic (competition contribution). In: Proc. TACAS. pp. 423-425. LNCS 9035, Springer (2015). https://doi.org/10.1007/978-3-662-46681$0 \quad 34$

37. Dietsch, D., Heizmann, M., Nutz, A., Schätzle, C., Schüssele, F.: Ultimate Taipan with symbolic interpretation and fluid abstractions (competition contribution). In: Proc. TACAS (2). LNCS 12079, Springer (2020)

38. Gadelha, M.Y.R., Monteiro, F.R., Cordeiro, L.C., Nicole, D.A.: Esbmc v6.0: Verifying $\mathrm{C}$ programs using $k$-induction and invariant inference (competition contribution). In: Proc. TACAS (3). pp. 209-213. LNCS 11429, Springer (2019). https://doi.org/10.1007/978-3-030-17502-3_15

39. Gadelha, M.Y., Ismail, H.I., Cordeiro, L.C.: Handling loops in bounded model checking of C programs via $k$-induction. Int. J. Softw. Tools Technol. Transf. 19(1), 97-114 (Feb 2017). https://doi.org/10.1007/s10009-015-0407-9

40. Gavrilenko, N., Ponce de León, H., Furbach, F., Heljanko, K., Meyer, R.: BMC for weak memory models: Relation analysis for compact SMT encodings. In: Proc. CAV. pp. 355-365. LNCS 11561, Springer (2019). https://doi.org/10.1007/978-3030-25540-4_19

41. Greitschus, M., Dietsch, D., Podelski, A.: Loop invariants from counterexamples. In: Proc. SAS. pp. 128-147. LNCS 10422, Springer (2017). https://doi.org/10.1007/9783-319-66706-5_7

42. Heizmann, M., Chen, Y.F., Dietsch, D., Greitschus, M., Hoenicke, J., Li, Y., Nutz, A., Musa, B., Schilling, C., Schindler, T., Podelski, A.: Ultimate Automizer and the search for perfect interpolants (competition contribution). In: Proc. TACAS (2). pp. 447-451. LNCS 10806, Springer (2018). https://doi.org/10.1007/978-3-31989963-3_30

43. Heizmann, M., Hoenicke, J., Podelski, A.: Software model checking for people who love automata. In: Proc. CAV. pp. 36-52. LNCS 8044, Springer (2013). https://doi.org/10.1007/978-3-642-39799-8_2

44. Holík, L., Kotoun, M., Peringer, P., Šoková, V., Trtík, M., Vojnar, T.: Predator shape analysis tool suite. In: Hardware and Software: Verification and Testing. pp. 202-209. LNCS 10028, Springer (2016). https://doi.org/10.1007/978-3-319-49052-6

45. Howar, F., Isberner, M., Merten, M., Steffen, B., Beyer, D.: The RERS grey-box challenge 2012: Analysis of event-condition-action systems. In: Proc. ISoLA. pp. 608-614. LNCS 7609, Springer (2012). https://doi.org/10.1007/978-3-642-34026$0 \_45$

46. Huisman, M., Klebanov, V., Monahan, R.: VerifyThis 2012: A program verification competition. STTT 17(6), 647-657 (2015). https://doi.org/10.1007/s10009-0150396-8

47. Inverso, O., Tomasco, E., Fischer, B., La Torre, S., Parlato, G.: Lazy-CSeq: A lazy sequentialization tool for C (competition contribution). In: Proc. TACAS. pp. 398401. LNCS 8413, Springer (2014). https://doi.org/10.1007/978-3-642-54862-8_29

48. Inverso, O., Trubiani, C.: Parallel and distributed bounded model checking of multi-threaded programs. In: Proc. PPoPP. ACM (2020)

49. Kahsai, T., Rümmer, P., Sanchez, H., Schäf, M.: JAYHorn: A framework for verifying Java programs. In: Proc. CAV. pp. 352-358. LNCS 9779, Springer (2016). https://doi.org/10.1007/978-3-319-41528-4_19 
50. Kahsai, T., Rümmer, P., Schäf, M.: JAYHorn: A Java model checker (competition contribution). In: Proc. TACAS (3). pp. 214-218. LNCS 11429, Springer (2019). https://doi.org/10.1007/978-3-030-17502-3_16

51. Kröning, D., Tautschnig, M.: CBMC: C bounded model checker (competition contribution). In: Proc. TACAS. pp. 389-391. LNCS 8413, Springer (2014). https://doi.org/10.1007/978-3-642-54862-8_26

52. Lauko, H., Ročkai, P., Barnat, J.: Symbolic computation via program transformation. In: Proc. ICTAC. pp. 313-332. Springer (2018). https://doi.org/10.1007/978-3-03002508-3 17

53. de Leon, H.P., Furbach, F., Heljanko, K., Meyer, R.: Dartagnan: Bounded model checking for weak memory models (competition contribution). In: Proc. TACAS (2). LNCS 12079, Springer (2020)

54. Luckow, K.S., Dimjasevic, M., Giannakopoulou, D., Howar, F., Isberner, M., Kahsai, T., Rakamaric, Z., Raman, V.: JDART: A dynamic symbolic analysis framework. In: Proc. TACAS. pp. 442-459. LNCSS 9636, Springer (2016). https://doi.org/10.1007/978-3-662-49674-9_26

55. Malík, V., Schrammel, P., Vojnar, T.: 2LS: Heap analysis and memory safety (competition contribution). In: Proc. TACAS (2). LNCS 12079, Springer (2020)

56. Mues, M., Howar, F.: JDART: Dynamic symbolic execution for Java bytecode (competition contribution). In: Proc. TACAS (2). LNCS 12079, Springer (2020)

57. Noller, Y., Păsăreanu, C.S., Le, X.B.D., Visser, W., Fromherz, A.: Symbolic PAThFinder for SV-COMP (competition contribution). In: Proc. TACAS (3). pp. 239-243. LNCS 11429, Springer (2019). https://doi.org/10.1007/978-3-03017502-3_21

58. Nutz, A., Dietsch, D., Mohamed, M.M., Podelski, A.: Ultimate KojaK with memory safety checks (competition contribution). In: Proc. TACAS. pp. 458-460. LNCS 9035, Springer (2015). https://doi.org/10.1007/978-3-662-46681-0_44

59. Peringer, P., Šoková, V., Vojnar, T.: PredatorHP revamped (not only) for intervalsized memory regions and memory reallocation (competition contribution). In: Proc. TACAS (2). LNCS 12079, Springer (2020)

60. Păsăreanu, C.S., Visser, W., Bushnell, D.H., Geldenhuys, J., Mehlitz, P.C., Rungta, N.: Symbolic PAThFinder: integrating symbolic execution with model checking for Java bytecode analysis. Autom. Software Eng. 20(3), 391-425 (2013). https://doi.org/10.1007/s10515-013-0122-2

61. Quiring, B., Manolios, P.: GaCAL: Conjecture-based verification (competition contribution). In: Proc. TACAS (2). LNCS 12079, Springer (2020)

62. Richter, C., Wehrheim, H.: PeSCo: Predicting sequential combinations of verifiers (competition contribution). In: Proc. TACAS (3). pp. 229-233. LNCS 11429, Springer (2019). https://doi.org/10.1007/978-3-030-17502-3_19

63. Rocha, H.O., Menezes, R., Cordeiro, L., Barreto, R.: Map2Check: Using symbolic execution and fuzzing (competition contribution). In: Proc. TACAS (2). LNCS 12079, Springer (2020)

64. Rocha, H., Barreto, R.S., Cordeiro, L.C.: Memory management test-case generation of $\mathrm{C}$ programs using bounded model checking. In: Proc. SEFM. pp. 251-267. LNCS 9276, Springer (2015). https://doi.org/10.1007/978-3-319-22969-0_18

65. Sharma, V., Hussein, S., Whalen, M., McCamant, S., Visser, W.: Java RAnger at SV-COMP 2020 (competition contribution). In: Proc. TACAS (2). LNCS 12079, Springer (2020)

66. Svejda, J., Berger, P., Katoen, J.P.: Interpretation-based violation witness validation for C: NitWit. In: Proc. TACAS. LNCS , Springer (2020) 
67. Visser, W., Geldenhuys, J.: Coastal: Combining concolic and fuzzing for Java (competition contribution). In: Proc. TACAS (2). LNCS 12079, Springer (2020)

68. Volkov, A.R., Mandrykin, M.U.: Predicate abstractions memory modeling method with separation into disjoint regions. Proceedings of the Institute for System Programming (ISPRAS) 29, 203-216 (2017). https://doi.org/10.15514/ISPRAS2017-29(4)-13

69. Wetzler, N., Heule, M.J.H., Jr., W.A.H.: Drat-Trim: Efficient checking and trimming using expressive clausal proofs. In: Proc. SAT. pp. 422-429. LNCS 8561, Springer (2014). https://doi.org/10.1007/978-3-319-09284-3_31

70. Yin, L., Dong, W., Liu, W., Li, Y., Wang, J.: Yogar-CBmc: Cвmc with scheduling constraint based abstraction refinement (competition contribution). In: Proc. TACAS. pp. 422-426. LNCS 10806, Springer (2018). https://doi.org/10.1007/9783-319-89963-3_25

71. Yin, L., Dong, W., Liu, W., Wang, J.: On scheduling constraint abstraction for multi-threaded program verification. IEEE Trans. Softw. Eng. (2018). https://doi.org/10.1109/TSE.2018.2864122

Open Access. This chapter is licensed under the terms of the Creative Commons Attribution 4.0 International License (http://creativecommons.org/licenses/by/4.0/), which permits use, sharing, adaptation, distribution, and reproduction in any medium or format, as long as you give appropriate credit to the original author(s) and the source, provide a link to the Creative Commons license and indicate if changes were made.

The images or other third party material in this chapter are included in the chapter's Creative Commons license, unless indicated otherwise in a credit line to the material. If material is not included in the chapter's Creative Commons license and your intended use is not permitted by statutory regulation or exceeds the permitted use, you will need to obtain permission directly from the copyright holder. 